\title{
Algorithm for Feature Extraction of Heart Sound Signal Based on Sym7 Wavelet
}

\author{
Guohua Zhang ${ }^{1, a}$, Zhongfan Yuan ${ }^{2, b}$ and Shixuan Liu ${ }^{1, c}$ \\ ${ }^{1}$ Shandong Provincial Key Laboratory of Ocean Environment Monitoring Technology, Shandong \\ Academy of Sciences Institute of Oceanographic Instrumentation, Qingdao 266001, China \\ ${ }^{2}$ School of Manufacturing Science and Engineering, Sichuan University, Chengdu 610065, China

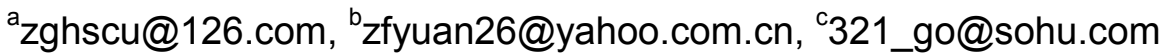

Keywords: heart sound signal, feature extraction, wavelet packet algorithm, class separability measure

\begin{abstract}
In order to extract pathological features of heart sound signal accurately, an algorithm for extracting the sub-band energy is developed based on the wavelet packet analysis. Through the spectrum analysis of heart sound signal, the sym 7 wavelet, with high energy concentration and good time localization, is taken as the mother function, and the best wavelet packet basis of heart sound signal is picked out. Then, various heart sound signals are decomposed into four levels and the wavelet packet coefficients of the best basis are obtained. According to the equal-value relation between wavelet packet coefficients and signal energy in time domain, the normalized sub-band energy of the best basis is extracted as the feature vector. The mean of class separability measure is 3.049 , which indicates that the algorithm is effective for feature extraction of heart sound signal.
\end{abstract}

\section{Introduction}

The cardiovascular disease is one of the diseases that threaten human health severely [1]. Heart sound recognition is to classify the diseases according to the characteristics of the heart sound. A.P. Yoganathan used fast fourier transform to analyze the first heart sound in normal man, and discovered that the heart sound signals usually are of non-stationary and time-varying characteristics due to the physiological, pathological or environmental effect [2]. The wavelet analysis has been widely used in the non-stationary signal analysis for its characteristic of time-frequency localization [3]. Yong Li carried out a time-frequency analysis of the heart sound signal by the continual wavelet transform, and discovered the process that the frequency component of heart sound signal changes with time [4]. Although the wavelet analysis is a kind of effective time-frequency analysis method, its decomposition scale is proportional to the signal frequency. Therefore, its high-frequency resolution is poor and its effective decomposition is only suitable for the low-frequency part of the signal. However, wavelet packet analysis can carry out decomposition for both low-frequency and high-frequency parts simultaneously, and determines the resolution in the different frequency band adaptively [3]. An algorithm for extracting the sub-band energy is developed based on the wavelet packet analysis. Various heart sound samples of normal men and heart disease patients were analyzed by using the algorithm, and the results indicate that the algorithm is effective for heart sound recognition.

\section{Heart Sound}

Heart sound contains much important diagnostic information, such as the heart function and mechanical condition of the aorta. Compared with the traditional electrocardiosignal, the pathology change caused by heart disease comes out earlier in heart sound [5]. The normal heart sound contains the first heart sound (s1), the second heart sound (s2), the third heart sound (s3) and the fourth heart sound (s4). S1 and s2 are audible, while s3 and s4 are very weak. Once the heart function appears exception, the heart sound will contain murmur besides s1 and s2 [6]. Many researchers attempted to 
analyze the heart sound with various methods. A. Djebbari analyzed heart sound with the short-time Fourier transformation, and discovered that the frequency components of s1 mainly concentrate in the $50 \sim 150 \mathrm{~Hz}$ scope, the frequency components of s2 mainly concentrate in the 50 200Hz scope [7]. B. El-Asir analyzed the heart sound with the time-frequency analysis method, and discovered that heart murmur of different heart diseases appears in the different time with different frequency [8].

\section{Algorithm of Wavelet Packet}

To a great extent, the mother function of wavelet packet influences analysis precision of signal. The time-frequency analysis of heart sound signal requires that the mother function has high energy concentration and good time localization. The Symlet wavelets, with high energy concentration and good time localization, are compactly supported and can extract the useful signals from transient events. After repeating test and contrast, the orthonormal sym 7 wavelet is finally taken as the mother function in this research.

On the basis of the wavelet multi-resolution analysis theory, record the scaling function $\phi(t)$ as $u_{0}(t)$ and the wavelet function $\psi(t)$ as $u_{1}(t)$, and then the function set $\left\{u_{n}(t)\right\}_{n \in Z}$ defined by Eq. 1 .

$$
\left\{\begin{array}{l}
u_{2 n}(t)=\sqrt{2} \sum_{k \in Z} h(k) u_{n}(2 t-k) \\
u_{2 n+1}(t)=\sqrt{2} \sum_{k \in Z} g(k) u_{n}(2 t-k)
\end{array}\right.
$$

Eq. 1 is called wavelet packet which determined by $u_{0}(t)=\phi(t)$, where $h(k)$ and $g(k)$ are CQF coefficients.

$$
U_{j}^{n}=\operatorname{clos}_{L^{2}(R)}\left\{2^{-j / 2} u_{n}\left(2^{-j} t-k\right), k \in Z\right\} \quad n=0,1,2, \cdots ; j \in Z
$$

Namely, the space $U_{j}^{n}$ is the closure of the subspace on $L^{2}(R)$, and the subspace is generated by the linear combination of the integral translation sequence of $u_{n}(t)$ at the scale $j$. The integral translation sequence $2^{-j / 2} u_{n}\left(2^{-j} t-k\right)$ is an orthogonal basis of space $U_{j}^{n}$.

For the nonnegative integer $n$,

$$
U_{j}^{2 n} \perp U_{j}^{2 n+1}, \quad U_{j-1}^{n}=U_{j}^{2 n} \oplus U_{j}^{2 n+1}, \quad j \in Z
$$

Thus at random scale $j$, the wavelet space $W_{j}$ can be decomposed as Eq.4.

$$
\begin{aligned}
& W_{j}=U_{j+1}^{2} \oplus U_{j+1}^{3} \\
& =U_{j+2}^{4} \oplus U_{j+2}^{5} \oplus U_{j+2}^{6} \oplus U_{j+2}^{7} \\
& \text { : } \\
& m=0,1, \cdots, 2^{k}-1 ; j, k=1,2, \cdots \\
& =U_{j+k}^{2^{k}} \oplus \cdots \oplus U_{j+k}^{2^{k}+m} \oplus \cdots \oplus U_{j+k}^{2^{k+1}-1}
\end{aligned}
$$

Space subdivision of wavelet packet is shown in Fig.1. The first row represents the frequency band of the original signal. The following rows represent the frequency bands at 4 decomposition scales, and column numbers represent the parameters of the frequency and location. At the first decomposition, divide the original signal frequency band into two, and obtained the high frequency sub-band $U_{1}^{1}$ and low frequency sub-band $U_{1}^{0}$ on the second row. Then, divide each sub-band into two again, making sure that each row cover the whole frequency band of the signal. Therefore, the wavelet packet analysis overcomes the limitation that the wavelet analysis only can carry out the decomposition in the space $V_{j}$, but can not in the space $W_{j}$. So the wavelet packet analysis is more suitable for the analysis and examination of the non-stationary signal.

Supposing $x(t)$ is the space function of $L^{2}(R)$, to its discrete sampling sequence $\{x(p)\}_{p=1,2, \cdots N}$, the algorithm of wavelet packet decomposition is expressed as Eq.5. 


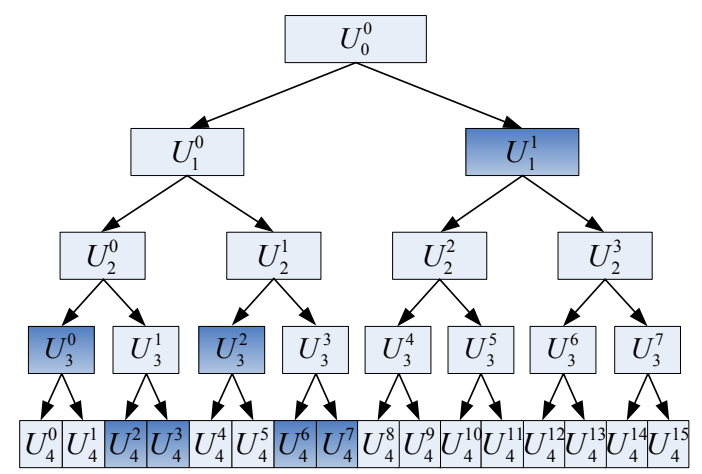

Fig.1 Space subdivision of wavelet packet and best basis of heart sound

$\left\{\begin{array}{l}C_{p}^{j, 2 n}=\sum_{k} h(k-2 p) C_{k}^{j+1, n} \\ C_{p}^{j, 2 n+1}=\sum_{k} g(k-2 p) C_{k}^{j+1, n}\end{array}\right.$

From the Eq.5, the wavelet packet decomposition materially is to decompose the signal into the different frequency bands through a group of CQF made up of LPF $h$ and HPF $g$ [9].

\section{Feature Extraction}

Parseval equation is Eq.6.

$\int_{-\infty}^{+\infty}|x(t)|^{2} d t=\sum_{k}\left|C_{j, k}\right|^{2}$

Where, wavelet transform coefficient $C_{j, k}$ is of the energy dimension, so it can be used in the energy analysis [10]. Heart sound signals of different heart diseases have the different energy distribution in each sub-band and thus the wavelet packet coefficients can be taken as the feature vectors of the heart sound signal.

Five kinds of heart sound signals, which may be confused easily during cardiophony, were taken as the sample set in this research. They are normal heart sound, splitting of first heart sound, splitting of second heart sound, soft first heart sound and loud second heart sound. For each kind of heart sound, ten samples were selected. The sampling frequency was set to $2000 \mathrm{~Hz}$. According to the sampling theorem, the Nyquist frequency is $1000 \mathrm{~Hz}$. Taking the sym 7 wavelet as the mother function to carry out four levels wavelet packets decomposition, the space subdivision of wavelet packet is shown in Fig.1. Through analysis of the samples, it can be discovered that the energy concentration of subspace $U_{4}^{0}$ is extremely low, and the energy concentration of subspace $U_{3}^{2}$ and $U_{1}^{1}$ are also low. To reduce the number of the wavelet packet basis, the further decomposition of $U_{3}^{0}, U_{3}^{2}$ and $U_{1}^{1}$ subspace is not necessary. The best wavelet packet basis is shown in the gray area of Fig. 1.

Supposing $E_{a, b}$ is the $b$ band energy of $a$ level, and then the feature vector is defined by Eq.7.

$T=\left(E_{3,0}, E_{4,2}, E_{4,3}, E_{3,2}, E_{4,6}, E_{4,7}, E_{1,1}\right)$

Supposing $E_{0}$ is the total energy of signal.

$E_{0}=E_{3,0}+E_{4,2}+E_{4,3}+E_{3,2}+E_{4,6}+E_{4,7}+E_{1,1}$

Then, the normalized feature vector is defined by Eq.9.

$$
T^{\prime}=\frac{T}{E_{0}}=\left(\frac{E_{3,0}}{E_{0}}, \frac{E_{4,2}}{E_{0}}, \frac{E_{4,3}}{E_{0}}, \cdots, \frac{E_{1,1}}{E_{0}}\right)
$$

Five kinds of heart sound signals and their normalized feature vectors after the wavelet packet transform are shown in Fig.2. The results indicate that the energy distribution is different in each frequency band for different heart sound signals, so it can provide the basis for the following pathology analysis. 

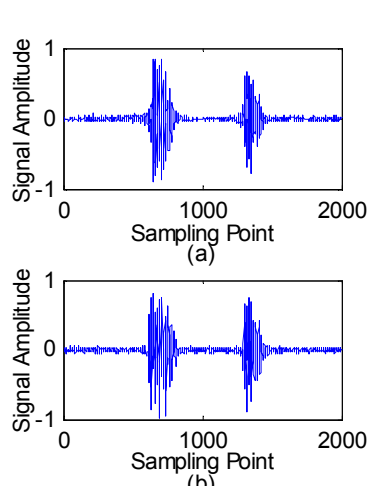

(b)
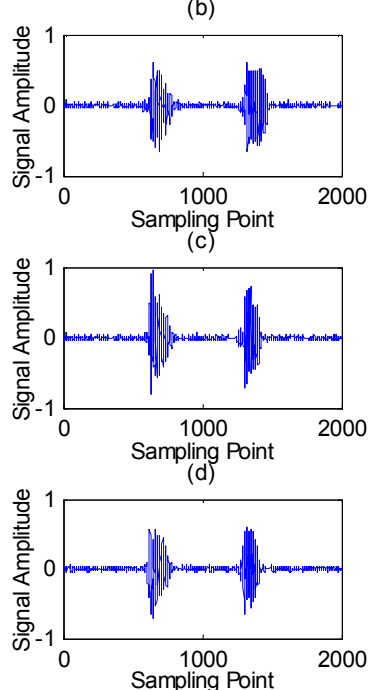

(e)
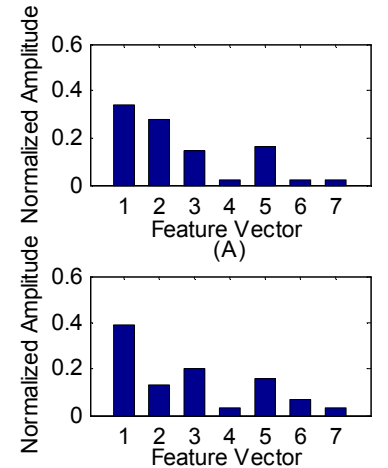

(B)

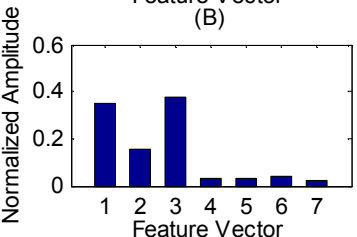

(C)

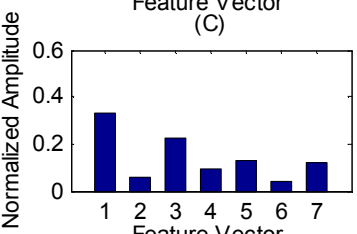

(D)

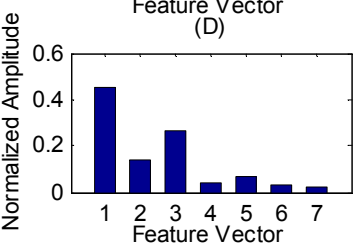

(E)

$\mathrm{A}(\mathrm{a})$ normal heart sound; $\mathrm{B}(\mathrm{b})$ splitting of first heart sound; C(c) splitting of second heart sound; D(d) soft first heart sound; $\mathrm{E}(\mathrm{e})$ loud second heart sound.

Fig.2 Heart sound signals and normalized feature vectors

Table 1 Separability measure of feature vector

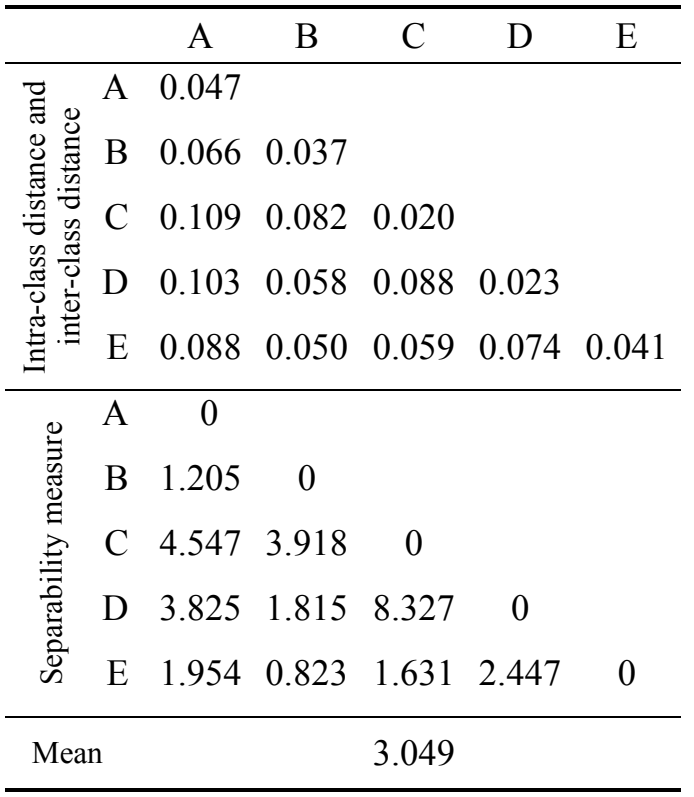

Note: A, normal heart sound; B, splitting of first heart sound; C, splitting of second heart sound; D, soft first heart sound; E, loud second heart sound.

\section{Estimate by the Class Separability Measure}

Regarding characteristic set $\left\{a_{i}, i=1,2, \cdots k\right\}$ having the identical characteristic, its intra-class distance is one of the important indexes of pattern separability. The square of the intra-class distance is defined by Eq. 10 .

$$
D^{2}\left(\left\{a_{i}\right\},\left\{a_{j}\right\}\right)=\frac{1}{k(k-1)} \sum_{i=1}^{k} \sum_{j=1}^{k} D^{2}\left(a_{i}, a_{j}\right)
$$

Regarding $\left\{a_{i}, i=1,2, \cdots k_{a}\right\}$ and $\left\{b_{i}, i=1,2, \cdots k_{b}\right\}$, where $a_{i} \in A$ and $b_{j} \in B$, the square of the inter-class distance between $A$ and $B$ is given by Eq.11.

$$
D^{2}\left(\left\{a_{i}\right\},\left\{b_{j}\right\}\right)=\frac{1}{k_{a} k_{b}} \sum_{i=1}^{k_{a}} \sum_{j=1}^{k_{b}} D^{2}\left(a_{i}, b_{j}\right)
$$

Integrate the Intra-class distance with inter-class distance, and thus the separability measure is defined by Eq.12.

$$
J_{A, B}=\frac{D^{2}\left(\left\{a_{i}\right\},\left\{b_{j}\right\}\right)}{D^{2}\left(\left\{a_{i}\right\},\left\{a_{i}\right\}\right)+D^{2}\left(\left\{b_{j}\right\},\left\{b_{j}\right\}\right)}
$$


$J_{A, B}$ can be used as an important index of separability measure between different classes. The bigger $J_{A, B}$ is, the better of the separability between class $A$ and class $B$, vice versa [10]. As shown in Table 1, the mean of the separability measures is 3.049 , which indicates that the algorithm can recognise the five kinds of heard sound effectively.

\section{Conclusion}

Heart sound is a typical non-stationary physiological signal, and heart sound recognition based on the wavelet analysis has become the new research direction in the field of heart sound diagnosis. Compared with the wavelet analysis, the wavelet packet analysis can obtain richer time-frequency local information, so it is more suitable for non-stationary signal analysis. From Fig.2, it can be seen that different kinds of heart sound samples were distinguished successfully by means of the scheme, and as shown in Table 1, the mean of separability measure is 3.049, which indicates that the algorithm is effective for heart sound recognition.

\section{Acknowledgement}

This work was supported by the Natural Science Foundation of Shandong Province (ZR2010HL056).

\section{References}

[1] Zhiru Bai, Yan Yang, Xiaorong Zeng. Research evolution of proteomics in cardiovascular disease. Advances in Cardiovascular Diseases. 29(2008)501-504.

[2] A.P. Yoganathan, R. Gupta, F.E. Udwadia, et al. Use of the fast fourier transform for frequency analysis of the first heart sound in normal man. Medical and Biological Engineering and Computing. 14(1976)69-73.

[3] J. Herold, R. Schroeder, F. Nasticzky, et al. Diagnosing aortic valve stenosis by correlation analysis of wavelet filtered heart sounds. Medical and Biological Engineering and Computing. 43(2005)451-456.

[4] Yong Li, Xiaorong Gao and Aiwen Guo. Time-frequency analysis of heart sounds based on continuous wavelet transform. Journal of Tsinghua University. 41(2001)77-80.

[5] Zhidong Zhao, Zhijin Zhao, Song Zhang, et al. A study on segmentation algorithm of heart sound. Space Medicine \& Medical Engineering. 17(2004)452-456.

[6] P.H. Stein, H.N. Sabbah, J.B. Lakier, et al. Frequency spectra of the first heart sound and of the aortic component of the second heart sound in patients with degenerated porcine bioprosthetic valves. The American Journal of Cardiology. 53(1984)557-561.

[7] A. Djebbari, F.B. Reguig. Short-time Fourier transform analysis of the phonocardiogram signal. The 7th IEEE International Conference on Electronics Circuits and Systems. (2000)844-847.

[8] B. El-Asir, L. Khadra, A.H. Al-Abbasi, et al. Time-frequency analysis of heart sounds. Digital Signal Processing Applications. (1996)553-558.

[9] Yi Liu, Caiming Zhang and Yuhua Peng, et al. The feature extraction and classification of lung sounds based on wavelet packet multiscale analysis. Chinese Journal of Computers. 29(2006) 769-777.

[10] Haiyan Zhang, Quan Zhou and Jindong Xia. Wavelet packet denoising and feature extraction for flaw echo signal in ultrasonic testing. Chinese Journal of Scientific Instrument. 27(2006)94-97. 